\title{
THE RESULTS OF OPERATION FOR ULNAR NEURITIS
}

\author{
MALCOLM F. MACNICOL
}

\author{
From the Department of Orthopaedic Surgery, University of Edinburgh
}

\begin{abstract}
The records of 110 cases of ulnar neuritis in 100 patients have been reviewed an average of 4.4 years after anterior transposition, or release of the aponeurosis. Seventy of the patients were reviewed personally. In over half the cases no precipitating cause was apparent. At operation the nerve was constricted by the flexor carpi ulnaris aponeurosis in fifty cases but in twenty-five no abnormal pathology was found. Recovery was greatest when operation was performed within three months of the onset of symptoms. In those cases where no abnormality was found, and those in which adhesions in the postcondylar groove involved the nerve, simple release was less effective than anterior transposition. It is therefore recommended that release be restricted to patients with a short history and with an obvious aponeurotic constriction unaccompanied by adhesions. Anterior transposition is the operation of choice where no abnormality is seen or where the nerve is dislocated, compressed or tethered proximal to the aponeurosis of flexor carpi ulnaris.
\end{abstract}

Tardy ulnar palsy was first described a century ago by Panas (1878) although the condition was appreciated even earlier (Platt 1926). Subsequently Broca and Mouchet (1899) reviewed a series of patients with post-traumatic ulnar neuritis, including one with anterior transposition, though the first report of this operation is attributed to Curtis (1898), who placed the nerve subcutaneously. Further reports followed in which the nerve was placed either subcutaneously or intramuscularly (Murphy 1916; Platt 1926).

In 1942 Learmonth described a submuscular anterior transposition, transferring the nerve deep to the forearm muscle mass so that it lay alongside the median nerve. Particular emphasis was laid on adequate release of the flexor carpi ulnaris aponeurosis distally and complete resection of the medial intermuscular septum proximally, to prevent the nerve from kinking at these sites. Deep transposition has become established in the management of ulnar neuritis, particularly in chronic or post-traumatic cases (Richards 1945; Gay and Love 1947; McGowan 1950; Vanderpool et al. 1968; Harrison and Nurick 1970; Levy and Apfelberg 1972; Seddon 1975); it seems to be less prone to postoperative complications than superficial placement (Gerl and Thorwirth 1974; Broudy, Leffert and Smith 1978).

Osborne (1957) reported briefly on the effect of simply dividing the aponeurotic arch which bridges the ulnar nerve 2 centimetres distal to the medial humeral epicondyle. He stated later (Osborne 1970) that simple release could give results comparable with those after anterior transposition of the nerve, so long as the symptoms were of relatively brief duration and an "acute on normal" nerve lesion existed. Feindel and Stratford (1958) called the compressing aponeurotic

arch the "cubital tunnel", and noted that a constricting band distal to the arch might also require division, as indeed may a fibrous band proximal to the arch (Ho and Marmor 1971).

No review comparing the results of anterior transposition with those of cubital tunnel release has been reported, although this comparison was recommended by Harrison and Nurick (1970). The indications for the two procedures differ (Vanderpool et al. 1968), but are often confused, even at operation. It was in the hope of resolving this confusion that the present review was undertaken. It consists of 110 cases of ulnar neuritis about half of which were treated by transposition and half by aponeurotic release.

\section{MATERIAL AND METHODS}

The case notes of 100 patients treated surgically for ulnar neuritis between the years 1965 and 1976 have been reviewed. Cases were excluded if the notes were inadequate. Seventy of these patients were reviewed personally at least one year after operation; the patients were examined before looking at the notes to see which operation had been performed. The information sought included the length of history and aetiology, the age, sex and occupation of the patient and hand dominance. Nerve conduction studies were seldom available and were therefore omitted. Patients with a history of rheumatoid arthritis or direct injury to the nerve were also excluded.

Ten cases were bilateral, so that information about 110 nerves was available. Fifty had been treated by anterior transposition; the medial intermuscular septum was incised, the flexor-pronator muscle mass reflected distally and the nerve rerouted deep to the muscle, which was then resutured to the epicondylar periosteum. Most of these patients wore a plaster cylinder with the elbow at a right angle for two or three weeks after operation. In fifty-eight arms the aponeurotic arch of the flexor carpi ulnaris was released, with or without neurolysis. The aponeurosis was not sutured deep to the nerve, as has been described recently by Osborne (1970). In two instances the only procedure carried out was release of the anconeus epitrochlearis muscle. 
Two anterior transpositions were revised because the nerve was not deep enough and symptoms had persisted; these were included in the review. Three patients treated by aponeurotic release also had persistent symptoms and needed anterior transposition; these, at review, were listed as anterior transpositions, but could be considered to be failed releases.

The length of follow-up after operation ranged from one to twelve years (mean 4.4 years). Men were affected twice as often as women. The age at presentation ranged from fifteen to eighty-four years with a mean of fifty-one years. The age distribution in decades is shown in Figure 1. The right arm was involved in fifty-nine of the 110 cases. Of the seventy patients seen personally, which included the ten bilateral cases, the dominant hand was involved twice as often as the other. Aetiology. In over half the cases no precipitating cause could be elicited. In fourteen cases a childhood fracture in the region of the elbow was reported, including a supracondylar fracture in five and a posterolateral fracture-dislocation in one. Osteoarthritis was radiologically evident in fifteen elbows, including five with a history of previous fracture. Thus a total of twenty-four ( 22 per cent) could be related to bony deformity, resulting from previous fracture or osteoarthritis. In none of these was a more recent injury reported; they therefore represented true tardy ulnar palsies.

Pressure on the nerve at the elbow, either during recumbency, after operation (Woltmann 1930) or from prolonged leaning on the elbow, accounted for the symptoms in a further seventeen (15 per cent). Four of these had dislocating ulnar nerves, and a further four cases with dislocating nerves were recorded in the group in which

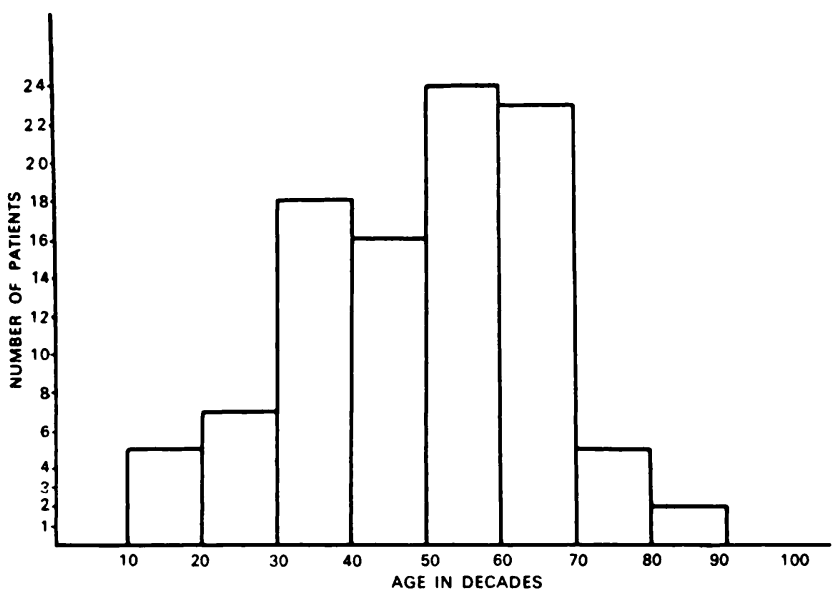

Fig. 1

Age distribution of 100 patients presenting with ulnar neuritis.

symptoms developed spontaneously. Vaguely remembered trauma accounted for symptoms in a further six cases, and there was a definite association with radiological cervical spondylosis in six instances, with carpal tunnel syndrome in a further four and with "tennis elbow" in two cases.

Findings at operation. Fifty of the nerves ( 46 per cent) showed the "Osborne lesion", the nerve being constricted by the aponeurosis of the flexor carpi ulnaris or "cubital tunnel"; in many instances it was also thickened proximal to the constriction. Adhesions, with or without thickening or narrowing of the nerve, were described as significant in 18 per cent. Probable muscle compression, either from the anconeus epitrochlearis or from a hypertrophied medial head of triceps, was present in 17 per cent. In eight instances the nerve was dislocated and in four cases a ganglion was found and thought to be compressing the ulnar nerve at the level of the elbow joint or in the cubital tunnel. The nerve appeared "stretched" over a post-traumatic deformity in two cases, and osteoarthritis was manifest in one patient by the presence of a loose body, and in another by a bony spicule. More than one lesion was considered significant in twenty instances, but in twenty-five ( 23 per cent) no lesion at all was found.
Table I. Objective assessment after operation

\begin{tabular}{|l|c|c|c|}
\hline & $\begin{array}{c}\text { None/ } \\
\text { minimal }\end{array}$ & Unchanged & Worse \\
\hline Symptoms in the hand & 1 & 0 & -1 \\
$\begin{array}{l}\text { (a) Weakness/clumsiness } \\
\text { (b) Paraesthesiae }\end{array}$ & 1 & 0 & -1 \\
$\begin{array}{l}\text { Discomfort in the elbow } \\
\begin{array}{l}\text { Objective sensory loss in the hand } \\
\text { (light touch, pinprick and } \\
\text { two-point discrimination) }\end{array}\end{array}$ & 1 & 0 & -1 \\
$\begin{array}{l}\text { Weakness of intrinsic muscles } \\
\begin{array}{l}\text { Weakness of long flexor muscles } \\
\text { (little finger flexor digitorum } \\
\text { profundus and flexor carpi } \\
\text { ulnaris) }\end{array}\end{array}$ & 1 & 0 & -1 \\
\hline
\end{tabular}

Assessment. The results of operation were assessed using a standard questionnaire and clinical examination. Symptoms and signs were graded on a point scale as shown in Table 1 . A highly successful outcome scores six points, although this does not necessarily mean that the arm is entirely symptom-free. Recovery was considered satisfactory if three or more points were scored, less than satisfactory if one to two points were scored, and frankly unsatisfactory if no points or a minus score was obtained. Muscle wasting was noted but was not included in the assessment.

Patient assessment. The seventy patients seen personally were asked to grade the success of their operation in relieving symptoms, using an arbitrary scale as follows: five points if they were extremely satisfied (no symptoms); four points if very satisfied (minimal and occasional symptoms); three points if fairly satisfied (intermittent and moderate symptoms); two points if disappointed (continuous moderate symptoms); one point if dissatisfied (unchanged postoperative symptoms); no points if most dissatisfied (symptoms worse since operation).

Questionnaires were sent to the thirty patients who did not present for personal review, but only seven replies were received and the information proved to be unreliable and difficult to interpret. An accurate evaluation of these patients was therefore impossible. Nevertheless, the recorded findings before and after operation have been included in the review.

\section{RESULTS}

Thirty-eight of the fifty arms in which anterior transposition was carried out were examined personally, and of the fifty-eight in which the aponeurosis alone was released forty-two were reviewed. Symptoms had been present before operation for an average of twelve months (one week to five years). The average length of history before anterior transposition (14.5 months) was slightly longer than before release (eleven months), but this difference was not statistically significant. Using the assessment scale described, the length of history was found to relate closely to the result. Figure 2 shows that the average result was unsatisfactory (less than two points) if the history exceeded one year. Even a history of less than three months was no guarantee of a completely successful result. Only five cases (two transpositions and three releases) scored six points, and in every instance the duration of symptoms before operation had been less than six months. In a further 
nine cases the muscle strength after operation was normal (MRC grade 5) in both the intrinsics and the long flexors; before operation only five of these had been normal, the others being strength 3 and 4 . All nine were well satisfied with the result although in every case there was still sensory loss on examination.

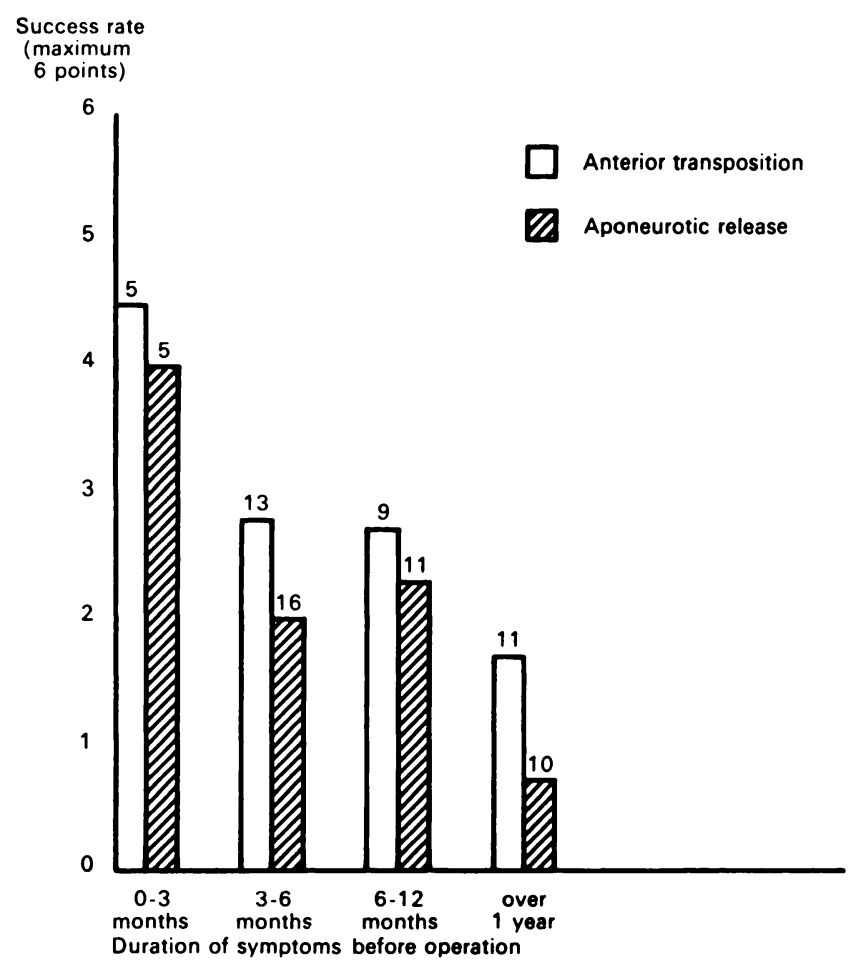

Fig. 2

Mean results plotted against time $(80 \mathrm{arms})$. The height of each column indicates the measure of success. The number of arms is shown at the head of each column.

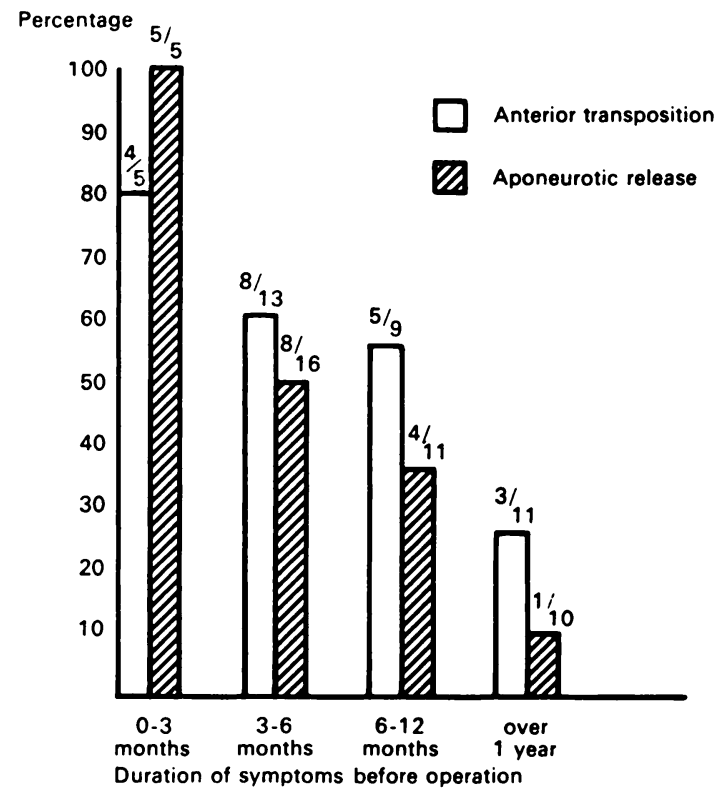

Fig. 3

Results plotted against time ( $80 \mathrm{arms})$. Percentage of arms in which a successful result was achieved. The actual numbers of successes and the total number in each group are shown at the head of each column.
Irrespective of the length of history, anterior transposition gave slightly greater improvement than simple release, although the difference was not statistically significant. In patients with symptoms of less than six months' duration the two operations gave comparable results; in those with a longer history anterior transposition was almost twice as likely to be successful. Only 10 per cent of patients with a history of more than one year attained a satisfactory result (three points or more) after aponeurotic release, and only 27 per cent after transposition (Fig. 3).

The relationship between the result and the discovery of abnormal pathology is shown in Table II. It is clear that in the anterior transpositions the proportion of poor results was not affected by the presence or absence of pathological changes. In those where aponeurotic release was performed, however, no less

Table II. Relationship of pathological findings to poor results. The figures refer to the number of arms

\begin{tabular}{|c|c|c|}
\hline Operation & $\begin{array}{l}\text { Abnormality } \\
\text { at operation }\end{array}$ & Poor result \\
\hline Anterior transposition & $\begin{array}{l}\text { Seen in } 31 \\
\text { Not seen in } 7\end{array}$ & $\begin{array}{l}5 \text { (16 per cent) } \\
1 \text { (14 per cent) }\end{array}$ \\
\hline Aponeurotic release & $\begin{array}{l}\text { Seen in } 30 \\
\text { Not seen in } 12\end{array}$ & $\begin{array}{l}4 \text { (13 per cent) } \\
8 \text { ( } 75 \text { per cent) }\end{array}$ \\
\hline
\end{tabular}

than 75 per cent of patients in whom no abnormality was found had a poor result.

Four of the poor results after transposition showed no muscle recovery; the same was true of nine of the twelve poor results after aponeurotic release. Muscle power often continued to improve after six months, and the long flexor muscles usually recovered more completely than the intrinsics. Nevertheless, there were only thirteen cases in which intrinsic muscle power had not returned to at least MRC grade 4. Patients in whom no muscle recovery occurred were, not surprisingly, dissatisfied with the result.

Sensory impairment was almost invariable; just over half the patients complained of this, but sensory deficit was found on examination in nearly all. Tenderness of the elbow, with or without a positive Tinel sign, was present in 33 per cent of patients after anterior transposition and in 31 per cent after aponeurotic release. The tenderness was usually localised to the postcondylar groove even after anterior transposition. Numbness over the point of the elbow (secondary to the incision) was noticed in over half the cases reviewed, but did not give rise to any concern.

Five patients were under twenty years of age; in four of these the result was excellent (five points or more) and these patients were well satisfied. One patient, with a history of a supracondylar fracture in childhood, achieved what seemed to be a satisfactory result, but he rated it poorly. Age had no obvious effect 


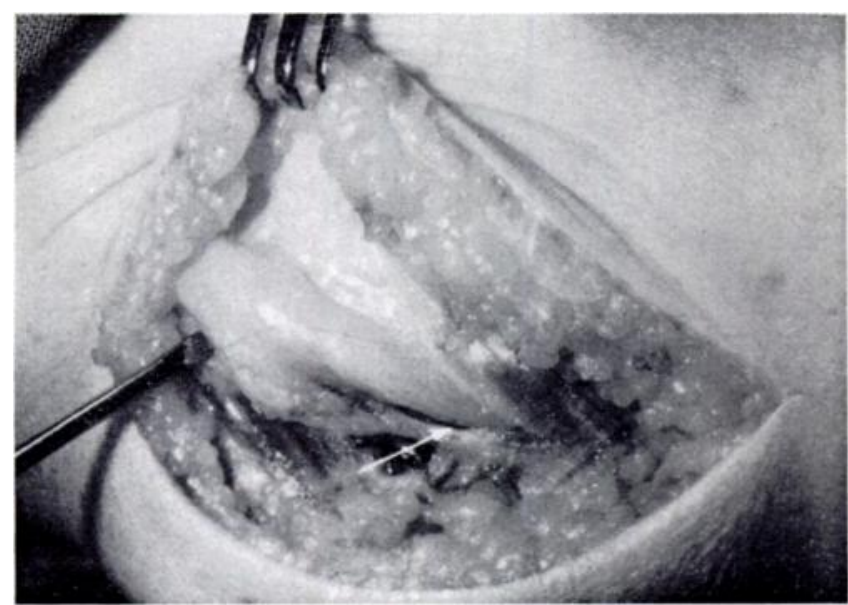

Fig. 4

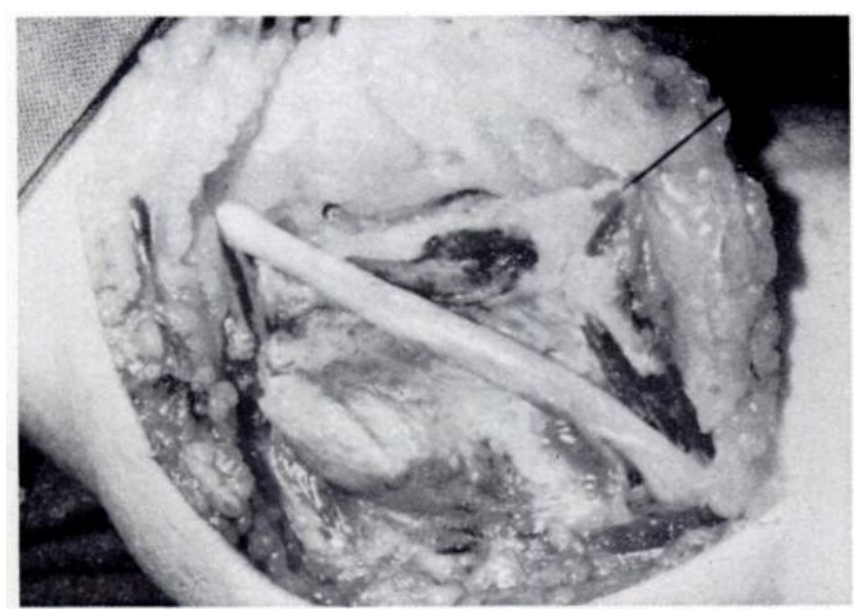

on the outcome in older age groups, nor did the patient's occupation.

Muscle release. In nineteen cases abnormal muscle compression was thought to have caused the ulnar neuritis; ten were due to an anconeus epitrochlearis muscle and nine to hypertrophy of the medial head of the triceps. Only nine of these cases were available for review. Five of these nine were examples of an anconeus epitrochlearis muscle; in them the nerve had been freed by muscle release and transposed anteriorly. In three of these five this resulted in an improvement of five points and a high degree of patient satisfaction; one was less than satisfactory, and at operation it had been noted that the nerve was thickened and bound down by adhesions; in the fifth case the only procedure was muscle incision which resulted in a normal hand (six points) three years later. In all four cases with hypertrophy of the triceps, the nerve was released from the muscle; in addition, one nerve had been transposed and the other three released distally at the cubital tunnel. One of these four cases, reviewed at ten years, had a poor result (one point) but at operation the nerve had been considerably thickened; the other three cases had a satisfactory outcome, comparable to that after anconeus epitrochlearis release.

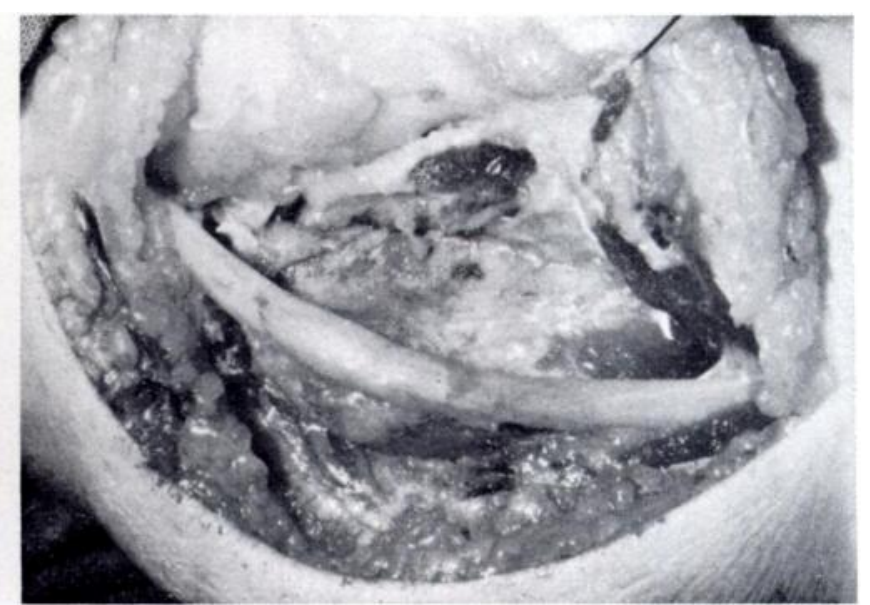

Fig. 5

Fig. 6

Anterior dislocation of the ulnar nerve. Figure 4-The nerve is riding over the prominence of the medial humeral epicondyle before passing under the aponeurotic arch of the flexor carpi ulnaris muscle origin (arrow). Figure 5-The medial intermuscular septum proximally and the flexor carpi ulnaris aponeurotic arch distally have been resected. The flexor-pronator muscle origin is being reflected from the epicondyle. Figure 6-The nerve is being transposed anteriorly under no tension deep to the flexor-pronator muscle mass. Note the groove over the medial humeral epicondyle produced by the nerve and the branch from the nerve supplying the flexor carpi ulnaris muscle.

Nerve dislocation. An anterior dislocation of the nerve had been found in eight cases, all of which were treated by anterior transposition (Figs. 4, 5 and 6). Six of these eight were examined. Four had regained normal muscle function and showed only slight loss of sensation, scoring an average of 4.5 points; the other two, in which significant adhesions were encountered at operation, had poor results, although the duration of symptoms before operation was not significantly different. In two patients the opposite nerve could be felt lying in a subluxed position.

Patient satisfaction. Patient satisfaction, assessed on a subjective scale from 5 (completely free of symptoms) to zero (symptoms worse than before operation), showed little difference between the two procedures. Anterior transposition scored an average of four points and aponeurotic release three and a half points, although anterior transposition was usually reserved for those with more florid or chronic symptoms. Approximately 75 per cent of patients rated the result as 3 (fairly satisfied) or better. Significant symptoms persisted in eighteen of the eighty arms reviewed (22.5 per cent); but in only nine cases (four after transposition and five after release) did patients feel that operation had been of no help (no points or one point). 


\section{DISCUSSION}

Ulnar neuritis secondary to compression, traction or friction of the nerve at the elbow has been recognised for a century. The usual presenting symptoms are numbness and tingling in the ulnar sensory distribution of the hand, and, less commonly, pain. Tenderness may be elicited in the region of the postcondylar groove, and pain may radiate down the ulnar aspect of the forearm. Muscle wasting may present early and, in patients with a long history, overt weakness of the hand becomes troublesome.

Normally the ulnar nerve elongates by almost 5 millimetres at the postcondylar groove during flexion (Apfelberg and Larson 1973) and it is able to glide freely at this site. Should the mechanics of the groove alter, either after a fracture or because of adhesions, then the nerve is no longer free to alter shape or position. Osborne (1957) drew attention to a potential site of compression where the nerve passes through the aponeurotic tunnel of the flexor carpi ulnaris. The nerve may also be compressed by fibrous bands just distal to this site (Feindel and Stratford 1958), by abnormal or hypertrophied muscle in the region of the elbow, and by ganglia or osteophytes (Vanderpool et al. 1968).

Pechan and Juliš (1975) have demonstrated in fresh cadavers that flexion of the elbow combined with extension of the wrist increases pressure in the ulnar nerve threefold. Furthermore, placing the hand behind the head results in intraneural pressure six times as high as in the relaxed nerve, and thus well above interstitial perfusion pressure (Fronek 1971). This pressure can be reduced significantly by releasing the flexor carpi ulnaris aponeurosis.

Stretching and compressive forces combine at the elbow to interfere with intraneural circulation in the ulnar nerve, resulting in hyperaemia and oedema (Weisl and Osborne 1964) with subsequent fibroblastic invasion. Once fibrosis is established, full relief of symptoms after decompression or transposition is unlikely. Hence the poor results when operation is long delayed. In this review it was clear that the presence of symptoms for more than a year before operation significantly diminished the chance of satisfactory recovery of the nerve. This recovery, with complete absence of symptoms and with normal sensation and power, is rarely attained if the history exceeds three months and if the symptoms have been continuous rather than intermittent. Hence the importance of early referral and early release of the nerve.

The two operations (transposition and release) cannot be accurately compared, because transposition is usually preferred for the more intractable cases. Nevertheless, the end results were more satisfactory after transposition, especially in those patients with histories exceeding six months. Poor results were especially evident in those cases where the cubital tunnel was released despite the fact that no definite abnormality was found on initial exploration, or where constriction of the nerve at the level of the tunnel was equivocal (Table II). While it is possible that symptoms in these cases may in part have arisen proximally, perhaps from cervical spondylosis, the relief afforded by anterior transposition in comparable instances was greater than that after simple release. The presence of adhesions in the region of the postcondylar groove also militates against a successful result.

The problem is that release, by its very simplicity, may encourage the inexperienced surgeon to limit himself to this procedure, particularly when no definite abnormality is encountered. When the history is short and aponeurotic constriction of the nerve is definite, then release gives good results; but with a longer history and if no abnormality is clearly evident, then transposition is recommended. If adhesions are present at the postcondylar groove, then again, despite the theoretical hazards, mobilisation of the nerve and anterior transposition result in an increased likelihood of success.

In patients with dislocation of the ulnar nerve (Platt 1926; Childress 1956) transposition is certainly the treatment of choice, since the nerve cannot be replaced in the postcondylar groove without undue tension. Where an abnormal muscle is considered to be the constricting agent, transposition again is recommended, although simple release has been shown in this review to be entirely successful on occasions. With anatomical or post-traumatic deformity transposition is widely accepted as the correct operation; providing care is taken to resect the medial intermuscular septum thoroughly and to ensure that the transposed nerve lies without kinking or tension, then the outcome should be satisfactory.

I wish to thank Mr J. Chalmers, Professor J. I. P. James, Mr D. W. Lamb and Mr W. M. McQuillan for their kindness in permitting me to review their patients; I am also grateful to Miss Helen Dingwall and Miss Yvonne Fraser for their secretarial help, and to Mr Michael Devlin for his skilful photography.

\section{REFERENCES}

Apfelberg, D. B., and Larson, S. J. (1973) Dynamic anatomy of the ulnar nerve at the elbow. Plastic and Reconstructive Surgery, 51, 76-81. Broca, A., and Mouchet, A. (1899) Complications nerveuses des fractures de l'extrémité inférieure de l'humérus. Revue de Chirurgie, 19, $701-706$.

Broudy, A. S., Leffert, R. D., and Smith, R. J. (1978) Technical problems with ulnar nerve transposition at the elbow: Findings and results of reoperation. Journal of Hand Surgery, 3, 85-89.

Childress, H. N. (1956) Recurrent ulnar-nerve dislocation at the elbow. Journal of Bone and Joint Surgery, 38-A, 978-984. 
Curtis, B. F. (1898) Traumatic ulnar neuritis-transplantation of the nerve. Journal of Nervous and Mental Disease, 25, $480-483$.

Feindel, W., and Stratford, J. (1958) Cubital tunnel compression in tardy ulnar palsy. Canadian Medical Association Journal, N.S. 78, 351-353.

Fronek, A. (1971) Isoconductometric estimation of effective capillary pressure in isolated hindlimb. American Journal of Physiology, 220, $1005-1008$.

Gay, J. R., and Love, J. G. (1947) Diagnosis and treatment of tardy paralysis of the ulnar nerve. Journal of Bone and Joint Surgery, 29-A, 1087-1097.

Gerl, A., and Thorwirth, V. (1974) Ergebnisse der Ulnarisverlagerung. Nachweis eines postoperativen Kompressions syndroms. Acta Neurochirurgica, 30, 227-246.

Harrison, M. J. G., and Nurick, S. (1970) Results of anterior transposition of the ulnar nerve for ulnar neuritis. British Medical Journal, $1,27-29$.

Ho, K. C., and Marmor, L. (1971) Entrapment of the ulnar nerve at the elbow. American Journal of Surgery, 121, $355-356$.

Learmonth, J. R. (1942) A technique for transplanting the ulnar nerve. Surgery, Gynecology and Obstetrics, 75, $792-793$.

Levy, D. M., and Apfelberg, D. B. (1972) Results of anterior transposition for ulnar neuropathy at the elbow. American Journal of Surgery, 123, 304-308.

McGowan, A. J. (1950) The results of transposition of the ulnar nerve for traumatic ulnar neuritis. Journal of Bone and Joint Surgery, 32-B, 293-301.

Murphy, J. B. (1916) Cicatricial fixation of the ulnar nerve from ancient cubitus valgus-release and transference to a new site. Clinics ofJohn B. Murphy, 5, 661-670.

Osborne, G. V. (1957) The surgical treatment of tardy ulnar neuritis. Journal of Bone and Joint Surgery, 39-B, 782.

Osborne, G. (1970) Compression neuritis of the ulnar nerve at the elbow. The Hand, 2, 10-13.

Panas, P. (1878) Sur une cause peu connue de paralysie du nerf cubital. Archives Générales de Médecine, VII serie, 2, 5-22.

Pechan, J., and Juliš, I. (1975) The pressure measurement in the ulnar nerve. A contribution to the pathophysiology of the cubital tunnel syndrome. Journal of Biomechanics, 8, 75-79.

Platt, H. (1926) The pathogenesis and treatment of traumatic neuritis of the ulnar nerve in the post condylar groove. British Journal of Surgery, 13, 409-431.

Richards, R. L. (1945) Traumatic ulnar neuritis-the results of anterior transposition of the ulnar nerve. Edinburgh Medical Journal, N.S. 52, $14-21$.

Seddon, H. J. (1975) Surgical Disorders of the Peripheral Nerves, pp. 114-117. Edinburgh and London: Churchill Livingstone.

Vanderpool, D. W., Chalmers, J., Lamb, D. W., and Whiston, T. B. (1968) Peripheral compression lesions of the ulnar nerve. Journal of Bone and Joint Surgery, 50-B, 792-803.

Weisl, H., and Osborne, G. V. (1964) The pathological changes in rats' nerves subjected to moderate compression. Journal of Bone and Joint Surgery, 46-B, 297-306.

Woltmann, H. W. (1930) Pressure as a factor in the development of neuritis of the ulnar and common peroneal nerves in bedridden patients. American Journal of the Medical Sciences, 179, 528-532. 